\title{
Parameterizing Compact and Extensible Compressor Models Using Orthogonal Distance Minimization
}

Xavier Llamas and Lars Eriksson

The self-archived pre-print version of this journal article is available at Linköping University Institutional Repository (DiVA):

http:/ / urn.kb.se/ resolve?urn=urn:nbn:se:liu:diva-136199

N.B.: When citing this work, cite the original publication.

Llamas, X., Eriksson, L., (2017), Parameterizing Compact and Extensible Compressor Models Using Orthogonal Distance Minimization, J ournal of engineering for gas turbines and power, 139(1), 012601-1-012601-10. https:/ / doi.org/ 10.1115/ 1.4034152

Original publication available at:

https:/ / doi.org/ 10.1115/ 1.4034152

Copyright: American Society of Mechanical Engineers (ASME)

http:// www.asme.org/ 


\section{Parameterizing compact and extensible compressor models using orthogonal distance minimization}

\author{
Xavier Llamas \\ Vehicular Systems, \\ Department of Electrical Engineering, \\ Linköping University, \\ Linköping SE-581 83, Sweden \\ e-mail: xavier.llamas.comellas@liu.se
}

\author{
Lars Eriksson \\ Vehicular Systems, \\ Department of Electrical Engineering, \\ Linköping University, \\ Linköping SE-581 83, Sweden \\ e-mail: lars.eriksson@liu.se
}

A complete and compact control oriented compressor model consisting of a mass flow submodel and an efficiency submodel is described. The final application of the model is a complete two-stroke mean value engine model which requires simulating the compressor operating at the low flow and low pressure ratio area. The model is based on previous research done for automotive-size compressors and it is shown to be general enough to adapt well to the characteristics of the marine-size compressors. A physics-based efficiency model allows, together with the mass flow model, extrapolating to low pressure ratios. The complexity of the model makes its parameterization a difficult task, hence a method to efficiently estimate the nineteen model parameters is proposed. The method computes analytic model gradients and uses them to minimize the orthogonal distances between the modeled speed lines and the measured points. The results of the parameter estimation are tested against nine different standard marine-size maps showing good agreement with the measured data. Furthermore, the results also show the importance of estimating the parameters of the mass flow and efficiency submodels at the same time to obtain an accurate model. The extrapolation capabilities to low load regions are also tested using low load measurements from an automotive-size compressor. It is shown that the model follows the measured efficiency trend down to low loads.

\section{Introduction}

The marine propulsion industry is facing new and more strict regulations on the engine exhaust emissions. For example, the Tier III regulations [1] have to be fulfilled for new vessels built from January 2016 on certain emission control areas. For the case of the low speed two-stroke marine engines, industry is developing and testing technologies that have potential to achieve such emission limits. One of these technologies is the Exhaust Gas Recirculation (EGR) that has been widely used in the automotive industry to reduce $\mathrm{NO}_{\mathrm{x}}$ formation during combustion and thus exhaust emissions.

Testing is required for the development of the EGR technology. However, performing tests on such big engines is limited mainly due to two reasons. First, there is a lack of available engines to perform such tests, the production numbers are much lower than the automotive case. The second issue is the very high economic cost required to perform tests in those large engines, mainly associated to fuel consumption. Due to these limitations, a reliable and fast dynamic engine model would be a valuable tool for the development of EGR systems and control strategies. A common approach for control oriented engine models is the Mean Value Engine Model (MVEM), which has the particularity that the model is based on average values of the engine cycle. For more information see e.g. [2-4]. Furthermore, [5] contains an overview of the targeted type of two-stroke engine as well as the current modeling status. Such engines contain several components that need to be modeled, and obtaining a reliable control oriented compressor model is one of the first challenges.

A compressor model consists of a mass flow model and an efficiency model, normally more emphasis is found for the mass flow-pressure ratio model in literature. Many different compressor models can be found in literature, in particular many different modeling approaches are investigated for automotive compressors, see e.g. [6,7]. For the much bigger compressors used for turbocharging the low speed twostroke engines there is less research done. Nevertheless some different models can be found in the literature. In [8] the compressor model is based on the fact that marine engines are loaded following the propeller law, and thus the compressor model has to be accurate only on the projected propeller curve in the compressor map. A more advanced model is presented in [9], where the compressor mass flow is modeled with exponential functions and the efficiency model is a 
quadratic function of mass flow and pressure ratio. Another modeling idea is found in [10], where the efficiency and the mass flow models are based in two dimensional fourth order polynomials of the pressure ratio and the compressor speed. An example that allows extrapolation of the compressor performance is described in [11], where the non-dimensional parameters are used to do this extrapolation. A final modeling approach, but applied to an axial compressor, is the rotated ellipse described in [12].

The main motivation here is to obtain a complete compressor model (mass flow and efficiency) that is compact and continuous, as well as not too complex so it can be used for control purposes. Furthermore, the model has to be capable of predicting the compressor performance at the low load area. This area is reached due to the interactions between the engine components during transient vessel maneuvering at low load. In particular it is observed that the pressure ratio may fall below unity when the auxiliary blower is engaged. The extrapolation has to follow the physical laws in order to be reliable. This is because this area is normally not covered in conventional compressor maps, and thus the extrapolation cannot be validated with the available measurements.

\subsection{Contributions}

A complete compressor model is described, which is capable of predicting mass flows and efficiencies at low loads using a mixed empirical and physical model. An automotivesize compressor measured at low loads is used to validate the assumptions taken for the physics-based efficiency model. The descriptive capabilities of the model are tested for a number of large compressor maps and shown to predict the measured points well.

Parameterizing a complex model is not a simple task, therefore a robust method for efficiently parameterizing the proposed compressor model is presented. The algorithm uses geometric properties of the model curves and exploits the analytic equations of the gradient to minimize the orthogonal distance between the model curves and the measured points.

\subsection{Outline}

General descriptions of the compressor maps and the corrected quantities are given in Section 2. An overview of the main characteristics of the compressor maps used in this study is presented in Section 3.

The proposed compressor model is described in detail in Section 4. Both mass flow submodel and efficiency submodel equations are introduced and motivated. After that, the proposed orthogonal projection method to parameterize the model is explained in Section 5.

Section 6 compares the model results to the measurements. It first compares the model to the extended measured automotive maps to assess its quality in terms of extrapolation capabilities and then it presents the results of the parameterized model to the marine-size compressor maps. Finally Section 7 contains the concluding remarks.

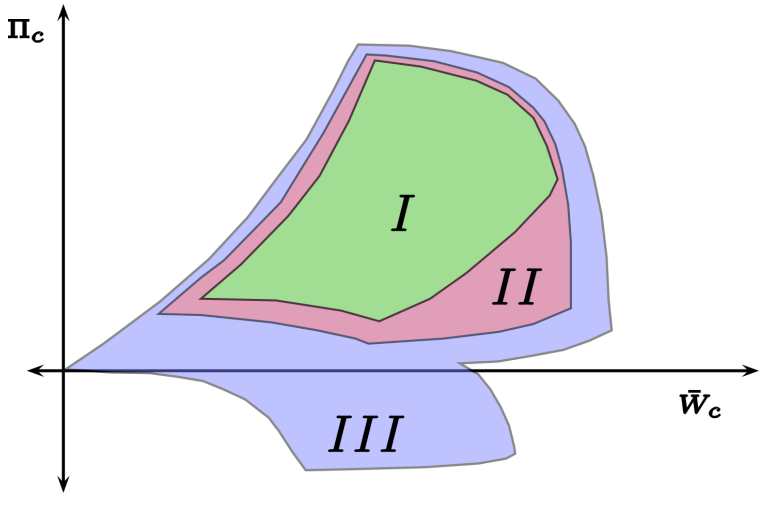

Fig. 1. Sketch of the three types of compressor maps: (I) Normal maps; (II) Full maps; (III) Extended maps

\section{Compressor Maps}

A compressor map contains the compressor performance characteristics for a number of measured operating points. Since the measurement of the compressor performance is relative to the inlet conditions, the maps are stored using corrected variables to cover different inlet conditions. These correction factors are based on dimensional analysis, a detailed explanation can be found in [13].

The compressor map is a graphical representation of the compressor performance showing stationary measurements of pressure ratio $\Pi_{c}=\frac{p_{02}}{p_{01}}$, efficiency $\eta_{c}$, corrected mass flow $\bar{W}_{c}$ and corrected speed $\bar{N}_{c}$. These corrected quantities are defined as in [14]

$$
\begin{aligned}
& \bar{W}_{c}=W_{c} \frac{\sqrt{T_{01} / T_{c, \text { ref }}}}{p_{01} / p_{c, \text { ref }}} \\
& \bar{N}_{c}=N_{c} \frac{1}{\sqrt{T_{01} / T_{c, \text { ref }}}}
\end{aligned}
$$

where $T_{c, r e f}$ and $p_{c, r e f}$ are the reference values.

Following the classification from [15], Figure 1 sketches the three types of compressor maps. Normal maps are the common ones provided by the manufacturers, they contain points with fairly high efficiencies and thus the pressure ratios are far above unity. Full maps contain points close to unity pressure ratio, limited to the discharge line of the gas stand. Extended maps contain points with pressure ratios below unity and even stand still operation.

For this study, all marine-size compressor maps used are normal maps. Unfortunately we have obtained neither full nor extended maps from a marine compressor, so the extrapolation capabilities cannot be completely validated. Instead, what is done is to rely on the research done for automotivesize compressors (see [15-17] among others) and assume that the main characteristics observed for the small size compressors will be similar for the large ones. Nevertheless a full automotive-size compressor map is used to see how well the complete model, mass flow and efficiency, can agree on the low load measurements. More information about the validation of the mass flow submodel at pressure ratios below unity 


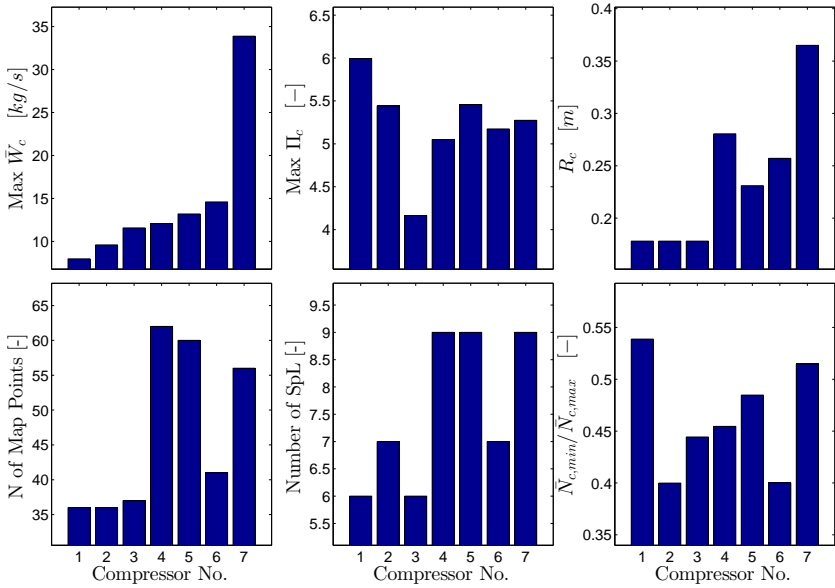

Fig. 2. General characteristics of seven of the marine-size compressor maps used.

using an extended automotive-size map can be found in [15].

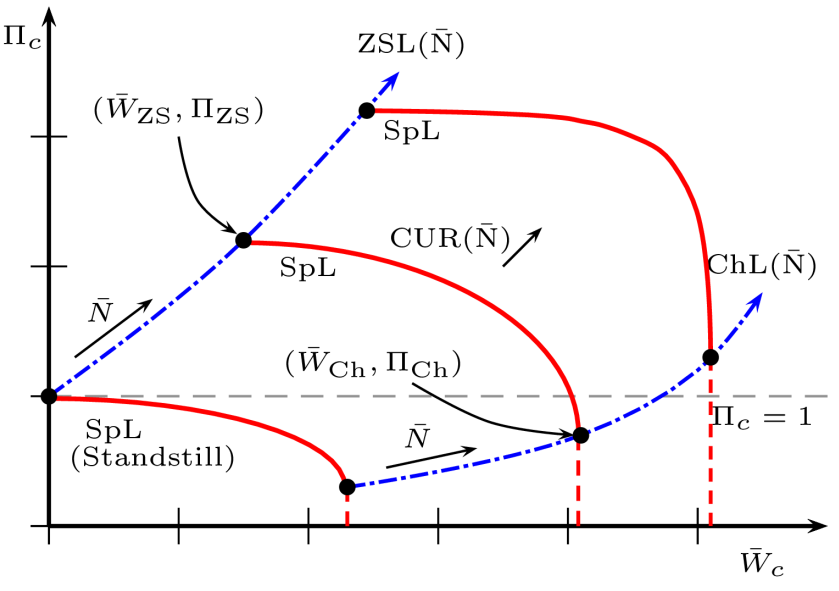

Fig. 3. Sketch of the main characteristics of the Ellipse model. The speed lines (SpL) are drawn in solid red, with its vertical extension in the choked area as dashed red line. The choking and zero slope lines are plotted in dashed-dotted blue lines. The dashed gray line situates where the pressure ratio equal to unity is. Note that the curvature increases with speed, and the lowest SpL corresponds to standstill.

\subsection{Compressor Mass Flow Model}

The mass flow-pressure ratio relation is modeled using the Ellipse model presented originally in [16] and its extension with the choking line in [15]. The model was developed so that it could cope with the data in a large database of automotive compressor maps of the three types depicted in Figure 1. The main reason to use these 15 parameters and not a simpler model with fewer parameters is its capacity to extrapolate to low pressure ratios and even stand still conditions. An overview of the model and its characteristics is presented in Figure 3.

The key parameters of the Ellipse model are the two asymptotes, the choking line $(\mathrm{ChL})$ and the zero slope Line (ZSL). The choking line is defined where the mass flow becomes choked, meaning that for a further decrease in pressure ratio there is no increase in mass flow. This line is defined by the choking corrected mass flow, $\bar{W}_{C h}$, and the choking pressure ratio, $\Pi_{C h}$ as can be seen in Figure 3. The mass flow is a function of compressor speed and it is defined as a power function for lower speeds, and an affine function for the higher speeds values. This switching behavior at $\bar{N}_{c, s}$ was observed and proposed in [16]. The equations are defined as

$$
\begin{aligned}
& \bar{W}_{C h}\left(\bar{N}_{c} \leq \bar{N}_{c, s}\right)=c_{1}+c_{2} \cdot \bar{N}_{c}^{c_{3}} \\
& \bar{W}_{C h}\left(\bar{N}_{c}>\bar{N}_{c, s}\right)=c_{4}+c_{5} \cdot \bar{N}_{c}
\end{aligned}
$$

where $\bar{N}_{c, s}$ is considered a parameter to be estimated. Note that this switching characteristic in the equations introduces a discontinuity in the model at the switching point.To avoid a jump in the choking mass flow when crossing the switching speed value, a continuity condition is introduced between the two equations. This is in fact a relation between the model parameters at $\bar{N}_{c, s}$, so it reduces the number of independent 
parameters from six to five. The dependent parameter is chosen to be $c_{4}$, and its value can be computed using

$$
c_{4}=c_{1}+c_{2} \bar{N}_{c, s}^{c_{3}}-c_{5} \bar{N}_{c, s}
$$

The choking pressure ratio line is defined as a single exponential function of corrected compressor speed. It has the following model

$$
\Pi_{C h}\left(\bar{N}_{c}\right)=c_{6}+c_{7} \cdot \bar{N}_{c}^{c_{8}}
$$

with three parameters to determine.

In the same way as the choke line, the zero slope line is defined by the zero slope mass flow, $\bar{W}_{Z S}$, and the zero slope pressure ratio, $\Pi_{Z S}$. Again, both parameters are modeled as functions of corrected compressor speed. The mathematical function for the zero slope line mass flow is

$$
\bar{W}_{Z S}\left(\bar{N}_{c}\right)=0+c_{9} \cdot \bar{N}_{c}^{c_{10}}
$$

where the numeric 0 emphasizes that the zero slope flow at standstill is zero. It has two parameters to determine. Moreover the zero slope pressure ratio is again modeled as an exponential function of compressor speed.

$$
\Pi_{Z S}(\bar{N})=1+c_{11} \cdot \bar{N}_{c}^{c_{12}}
$$

with two parameters to determine. The numeric 1 in the equation sets the $\Pi_{Z S}$ equal to 1 at standstill.

Given a compressor corrected speed, the choking point $\left(\bar{W}_{C h}, \Pi_{C h}\right)$ and the zero slope point $\left(\bar{W}_{Z S}, \Pi_{Z S}\right)$, are determined. Thus the quarter of ellipse that joins these two points is in implicit form, $F\left(\bar{W}_{c}, \Pi_{c}\right)=0$, and $F$ is formulated as

$$
F\left(\bar{W}_{c}, \Pi_{c}\right)=\left(\frac{\bar{W}_{c}-\bar{W}_{Z S}}{\bar{W}_{C h}-\bar{W}_{Z S}}\right)^{C U R}+\left(\frac{\Pi_{c}-\Pi_{C h}}{\Pi_{Z S}-\Pi_{C h}}\right)^{C U R}-1
$$

where $C U R$ is the curvature of the ellipse. Usually the numeric value of $C U R$ starts around 2 and increases with corrected speed, see Figure 3. Thus in strict mathematical terms the model is not a pure ellipse, a more suitable name would be a hyper-ellipse since in general $C U R>2$. The curvature is shaped using the following power function

$$
\operatorname{CUR}(\bar{N})=c_{13}+c_{14} \cdot \bar{N}_{c}^{c_{15}}
$$

with three parameters to be estimated. In total the mass flow model consists of fifteen parameters.

The implicit version of the model in (10) is described because it is suitable to be used for the model parameterization which is explained in Section 5. However in the final simulation framework, the model is implemented as shown in (3). Hence the model is expressed as function of pressure ratio, then the explicit equation of mass flow is

$$
\bar{W}_{c}=\bar{W}_{Z S}+\left(\bar{W}_{C h}-\bar{W}_{Z S}\right) \cdot\left(\left(\frac{\Pi_{c}-\Pi_{C h}}{\Pi_{Z S}-\Pi_{C h}}\right)^{C U R}-1\right)^{\frac{1}{C U R}}
$$

Note that below the choking point, the model becomes a vertical line as it can be seen in Figure 3. Together with the choked flow for pressure ratios below $\Pi_{C h}$, the model can be expressed in explicit form as

$$
\bar{W}_{c}\left(\Pi_{c}, \bar{N}_{c}\right)= \begin{cases}\text { equation (12) } & \text { if } \Pi_{c} \geq \Pi_{C h} \\ \bar{W}_{C h} & \text { if } \Pi_{c}<\Pi_{C h}\end{cases}
$$

Finally, it is important to point out that the surge area is not modeled. The model as proposed in [15] contains a third order polynomial to model flows smaller than the zero slope flow at the current SpL. Unfortunately, there are no measured points that would let identify the model parameters in the normal marine-size compressor maps used in this study. Note that if surge simulations are required, it is possible to invert (13) to predict pressure ratio given the mass flow through the compressor.

\subsection{Compressor Efficiency Model}

The main requirement on the efficiency model is that it has to be able to extrapolate to low pressure ratios. It is also of importance that this extrapolation follows the physical laws of centrifugal compressors, so it is not only the extrapolation of an empirical function that can predict the measured points. This physical requirement increases the model reliability since as previously said, there is no measurement data available at low loads for the marine-size compressors.

Many compressor efficiency models can be found in the literature, however this number is greatly reduced if it has to follow these two main requirements. A compact approach, based on the compressor dimensionless parameters, that fulfills these requirements is developed and described in [18]. A similar reasoning but with another formulation is applied in [17] and further studied in [19]. A more in depth analysis of the physics involved in the previous cited models can be found in [20]. These results are developed in [21,22] into a more advanced 1D model. This model is, however, more oriented to predict the compressor performance given geometrical and design parameters. A OD model is proposed here, based on the work presented in [17], which has the advantage to require only four parameters that need to be estimated from measurement data. The derivation of the model equations is reproduced here for completeness.

The compressor "total to total" isentropic efficiency is defined as, i.e. the work required for an ideal adiabatic compression divided by the actual work required to achieve the same pressure increase

$$
\eta_{c}=\frac{\text { isentropic work }}{\text { actual work }}=\frac{h_{02_{s}}-h_{01}}{h_{02}-h_{01}}=\frac{\Delta h_{i s}}{\Delta h_{a c t}}
$$


following the reasoning in [13] and [23]. The specific energy consumed by the ideal adiabatic compression process from an initial state with pressure $p_{01}$ and temperature $T_{01}$ to a higher pressure state, $p_{02}$ is defined as

$$
\Delta h_{i s}=c_{p} T_{01}\left[\left(\frac{p_{02}}{p_{01}}\right)^{\frac{\gamma-1}{\gamma}}-1\right]
$$

where $c_{p}$ is the specific heat at constant pressure, and $\gamma$ is the ratio of specific heats.

The actual specific energy required to preform such pressure increase is derived using Euler's equation applied to the compressor speed triangles, see e.g. [24]. Moreover the same result can be obtained using rothalpy conservation and applying the rothalpy definition at the inlet and outlet of the radial compressor. Two main assumptions need to be done about the flow characteristics to obtain the final equation. The first assumption is that the air entering the impeller has no tangential velocity. The second is that the flow is only accelerated and not compressed in the impeller, thus at the impeller's outlet, the density is the same as in the inlet. This last assumption is shown not to hold according to the investigation carried out in [22], however it is taken here to agree with the $0 \mathrm{D}$ formulation and to keep the model complexity low. Using these two assumptions and the geometric definitions of the speed triangles on the Euler's equation the following relation is obtained

$$
\Delta h_{\text {act }}=U_{2}^{2}-\frac{U_{2} \cdot \operatorname{cotan}\left(\beta_{2}\right)}{\rho_{01} \cdot S_{\text {out }}} W_{c}
$$

where $U_{2}$ is the blade tip speed, $S_{\text {out }}$ is the cross section of the flow at the impeller's outlet, $\rho_{01}$ is the inlet density and $\beta_{2}$ is the outlet blade angle. Note that the expression is not given for corrected mass flow $\left(\bar{W}_{c}\right)$, but it can be rewritten using (2). One can notice that, given a fixed compressor speed, (16) is a linear function of compressor mass flow, if the previous assumptions hold. This is the key fact for the simplicity of the efficiency model used here. The performance of this linear description is shown in [17] and [19] for several automotive-size compressors.

For the marine-size compressors, the actual enthalpy gain is computed with the map measured variables and using both (14) (15). Figure 4 shows the computed actual enthalpy gain against corrected mass flow for two different marinesize compressors. In the left plot, the linearity assumption is quite accurate. However for the right plot, the enthalpy is not as linear when it approaches the choking region and specially for the high speed lines. It is important to note that for most of the compressor maps used, there is a lack of relatively low mass flow measurement points for the higher SpLs. In other words, the majority of the points are measured relatively close to the choking limit. The practical implication can be seen in the right plot of Figure 4, where the slope for the last $\mathrm{SpL}$ is greatly influenced by the fast drop in enthalpy probably due to choking. The lack of points to the left
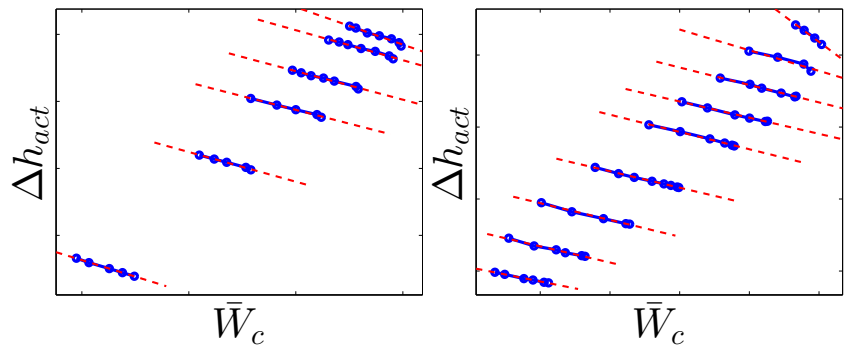

Fig. 4. Actual compression specific energy plotted against corrected mass flow for two different compressors. The measured data is plotted in blue dots connected with solid lines, and the linear fit is plotted in dashed red lines. Data corresponds to marine-size normal maps. A good agreement with the linear assumption can be observed, as well as slight deviations for the high SpL close to the choke region.

of this SpL may force the parameterization to choose a too steep line. This is one of the reasons why a total estimation of the whole model will be important, since it will introduce a trade-off of model agreement for all SpLs at the same time.

The same type of plot is depicted in Figure 5, this time for the full automotive-size map. This map has more measured points, and thus has a larger range of mass flow measurement for each $\mathrm{SpL}$, which is very helpful to validate the linear assumption. One can observe in the left plot that the linear relation holds quite well in general. Nevertheless the enthalpy drop for high flows is still observed. This phenomena can be explained by the choking effect, which is also observed in Figure 4. On the other hand, larger deviations are observed in the right plot, which corresponds to a zoomed view of the first six SpL of the automotive compressor. The reason for these deviations can be explained with the research done in [20]. In the low mass flow region, the deviations are accounted for the slip factor and the disc friction. This is observed mostly at lower SPLs since in absolute terms the losses represent a larger portion of the overall specific energy.

Following the previous reasoning, and taking into consideration what is presented in [20], a linear model is not the most accurate physical way to extrapolate the efficiency to the low load area. However, it follows the general trend of the efficiency trajectory at low loads. Moreover it has a very well balanced model in terms of accuracy vs. complexity, which is an important metric for control oriented models. Hence the following linear model is chosen to describe the actual enthalpy gain as function of mass flow

$$
\Delta h_{a c t}=b\left(\bar{N}_{c}\right)-a\left(\bar{N}_{c}\right) \cdot \bar{W}_{c}
$$

where the two parameters $a$ and $b$ are modeled as quadratic functions of compressor speed. These quadratic relations are proposed in [19]. Furthermore, these quadratic polynomials are forced to output 0 at standstill to follow (16), where both terms depend on compressor speed and will be zero at 

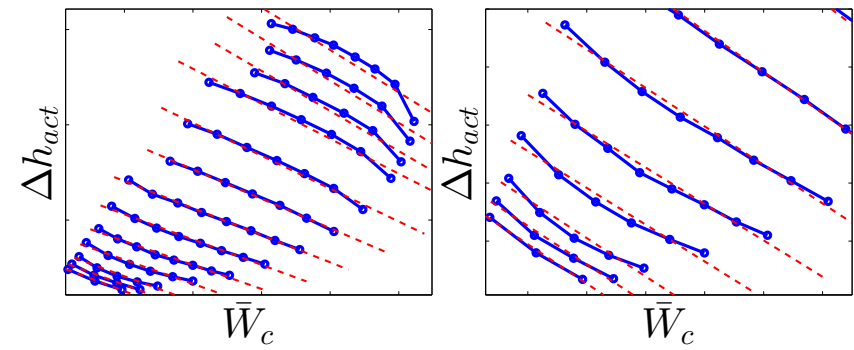

Fig. 5. Actual compression specific energy plotted against corrected mass flow for the full automotive map. The measured data is plotted in blue dots connected with solid lines, and the linear fit is depicted in dashed red lines. The right plot is a zoomed view of the first six SpLs seen in the left plot. More deviations from the linear assumption can be seen, in particular at low pressure ratios and for the higher SpLs.

standstill. The definitions of the quadratic polynomials are

$$
\begin{aligned}
& a\left(\bar{N}_{c}\right)=0+c_{16} \cdot \bar{N}_{c}+c_{17} \cdot \bar{N}_{c}^{2} \\
& b\left(\bar{N}_{c}\right)=0+c_{18} \cdot \bar{N}_{c}+c_{19} \cdot \bar{N}_{c}^{2}
\end{aligned}
$$

Moreover to ensure a smooth extrapolation of the efficiency down to standstill, a couple of conditions are imposed to the parameters. The parameter $c_{19}$ is forced to be positive, since $b\left(\bar{N}_{c}\right)$ identifies it directly to $U_{2}^{2}$ according to (16). Furthermore, the parameter $c_{18}$ should be ideally zero since $U_{2}^{2}$ only contains the quadratic term. Nevertheless it is included to increase the model flexibility. It is forced to be positive to avoid crossing zero for positive values of compressor speed, which would let to lose the smoothness of the efficiency extrapolation.

When used for simulation, the model has to follow the input-output relation described in (3). It is important to note here that the efficiency model requires both the mass flow and the pressure ratio values, as well as the compressor speed value to compute the efficiency. This means that the mass flow model described in Section 4.1 needs to be used inside the efficiency model. In other words, given $\left(\Pi_{c}, \bar{N}_{c}\right)$, $\bar{W}_{c}$ is computed using (13). Then given $\left(\bar{W}_{c}, \Pi_{c}, \bar{N}_{c}\right)$ the efficiency can be computed. This fact has a major influence because one should not forget that the errors from the mass flow model will affect the efficiency model output.

\section{Compressor Model Parametrization}

Given the overall compressor model described in the previous Section, the next step is to find the parameters that describe the measured data best. This is not a trivial task, especially because the model is highly nonlinear and thus solving the least-squares problem is not a straightforward task. This Section describes a parameterization strategy that exploits the models mathematical properties.

\subsection{Initial Guess}

Finding a good initial guess of the parameters is a very important issue for the solver performance in a nonlinear optimization problem. First of all the choke and zero slope lines shown in Figure 3 are found by visual inspection. Given the measured data one can have a rough idea where both lines are situated in the $\Pi_{c}-\bar{W}_{c}$ space. Then separate least squares problems are solved to parameterize the functions (4) (5) (7) (8) (9) to the estimated choke and zero slope lines. After that and keeping the already obtained parameters fixed, the curvature $(C U R)$ values are estimated solving a least-squares problem for each $\mathrm{SpL}$ of the compressor map. Then the function described in (11) is fitted to the estimated $C U R$ values as function of corrected compressor speed.

The remaining four parameters from the efficiency model (18) and (19) are obtained by solving several independent linear least-squares problems to find $a$ and $b$ for all $\mathrm{SpL}$. A visual example can be seen in Figure 4, where the linear model is plotted in a dashed red line. Then the polynomial functions are estimated to the previous estimated values of $a$ and $b$ for each speed value using least-squares.

\subsection{Mass flow orthogonal parametrization}

The initial parameters are mostly obtained by visual inspection of the compressor map, so the fit can be greatly improved if all the parameters are estimated at the same time. In order to avoid increasing the problem complexity too much the total estimation is done first for the mass flow submodel alone.

Typical least-squares problems penalize the error of the dependent variable, see e.g. [25] for a complete description. For this particular problem this means having to work with the explicit version of the mass flow model (12), which introduces problems due to the infinite slope of the model close to the zero slope point. To avoid this, the squared orthogonal distance from the measured point to the model curve is minimized instead. This approach has proven to be useful when dealing with curves and surfaces. For example, different results between the common least squares approach and the orthogonal distance minimization for fitting circles and ellipses is shown in [26] and [27]. Furthermore, a complete algorithm that minimizes the sum of the squared orthogonal distances is described in [28]. In this study the idea of minimizing the distance remains the same as the cited papers, however the implementation relies on regular least squares algorithms not implemented by the authors. Instead, what is proposed is the method to compute the squared distances to be minimized.

The orthogonal least squares algorithm is implemented in two layers. The outer layer is still to minimize the sum of the squares, however in this case the squared variable is the orthogonal distance. To compute the distances first the orthogonal projection needs to be solved given the current parameters.

The first step is thus to find the orthogonal projection from each measurement point the the model curve. To do that, the mathematical structure of the model can be used to 


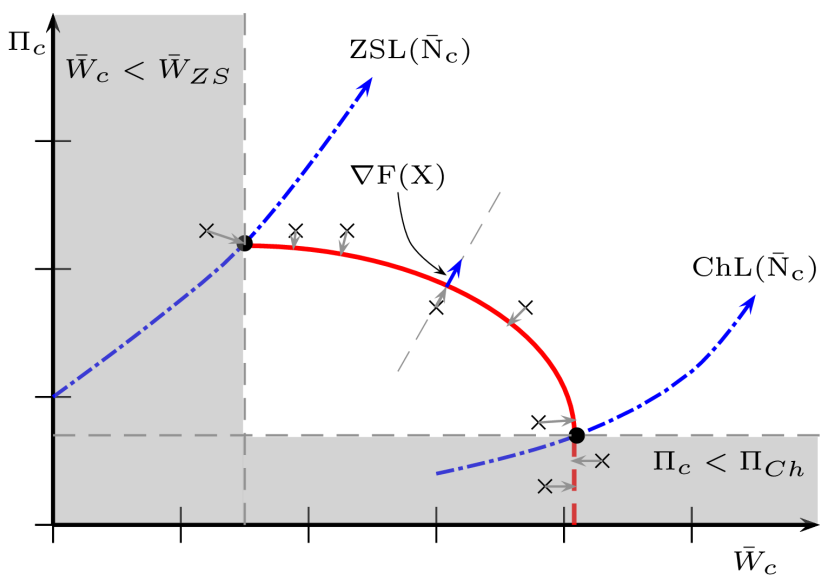

Fig. 6. Orthogonal projection representation of the SpL measured points (crosses) to the modeled SpL curve in solid red. The gradient of the implicit model equation is used to find the orthogonal direction. The shaded area represents the zones where the orthogonal projection cannot be computed.

simplify the projection. Note that given a speed value $\left(\bar{N}_{c}\right)$, the ellipse curve is fully defined. The choking and the zero slope coordinates in the $\Pi_{c}-\bar{W}_{c}$ plane are fixed, and these two points define the beginning and the end of the modeled curve. Moreover the map measurements are done also for constant speed values. So the orthogonal projection can be divided into $n$ subproblems, where $n$ is the number of measured speed lines. A sketch of this observation is shown in Figure 6 where the orthogonal projections from each measurement point corresponding to the same measured speed to the modeled SpL curve can be seen.

In order to find the orthogonal intersection geometrically, a system of equations need to be solved. Using mathematical geometry theory in two dimensions the equations can be derived as

$$
\left\{\begin{array}{l}
F(X)=0 \\
\vec{n}_{i}(X) \cdot\left(X_{i}-Y_{i}\right)+\vec{n}_{j}(X) \cdot\left(X_{j}-Y_{j}\right)=0
\end{array}\right.
$$

where

$$
\begin{aligned}
& X=\left(\bar{W}_{c, \text { int }}, \Pi_{c, \text { int }}\right)^{T} \\
& Y=\left(\bar{W}_{c, \text { meas }}, \Pi_{c, \text { meas }}\right)^{T}
\end{aligned}
$$

where the first equation ensures that the intersection point $\left(\bar{W}_{c, \text { int }}, \Pi_{c, \text { int }}\right)$, which is the unknown, belongs to the modeled SpL curve, (10). The second equation ensures that it also belongs to the perpendicular line to the ellipse at the intersection point that goes through the measurement point $\left(\bar{W}_{c, \text { meas }}, \Pi_{c, \text { meas }}\right)$. For that to hold, the normal vector of the line $(\vec{n})$ has to be perpendicular to the ellipse gradient at the intersection point. With this information the normal vector is $\vec{n}$ defined as

$$
\vec{n}(X)=\perp \nabla F(X)=\left[\frac{\mathrm{d} F(X)}{\mathrm{d} \Pi_{c}},-\frac{\mathrm{d} F(X)}{\mathrm{d} \bar{W}_{c}}\right]^{T}
$$

The analytic expression of the gradients can be found in Appendix A. Given the intersection point, the square of the orthogonal distance is computed as

$$
\begin{aligned}
d_{n} & =\left(\frac{\Delta \bar{W}_{c, n}}{\text { max } \bar{W}_{c, \text { meas }}}\right)^{2}+\left(\frac{\Delta \Pi_{c, n}}{\max \Pi_{c, \text { meas }}}\right)^{2} \\
\Delta \bar{W}_{c, n} & =\bar{W}_{c, \text { inter }, n}-\bar{W}_{c, \text { meas }, n} \\
\Delta \Pi_{c, n} & =\Pi_{c, \text { inter }, n}-\Pi_{c, \text { meas }, n}
\end{aligned}
$$

where $n$ runs over all measured points, and the distances are weighted by the maximum measured values at the current SpL.

The projection equations have a particularity, which is, that given the particular equation of the ellipse, the orthogonal distance can only be computed from measured points in a certain area of the $\Pi_{c}-\bar{W}_{c}$ plane. Figure 6 shows a shaded area where (20) has no solution. This is treated following two distinct cases. First for the points that have a mass flow measurement smaller than the zero slope point for that particular $\operatorname{SpL}\left(\bar{W}_{c, \text { meas }}<\bar{W}_{Z S}\right)$, the projected point is determined to be $\left(\bar{W}_{Z S}, \Pi_{Z S}\right)$, as Figure 20 shows. This is a simple assumption but it overcomes the problem and if the point is not too far away the distance is not much larger than the one computed for the other points. Moreover, for normal maps it is not common to have measured points in that area.

The second case, which is related to measurement points already being in the choking area $\left(\Pi_{c, \text { meas }}<\Pi_{c h}\right)$, arises much more often due to the fact that normal maps have quite steep $\mathrm{SpL}$ in the high mass flow region. The algorithm will to try to exploit the model geometry to obtain the best fit, meaning that it can push up the choking line if the parameters are not constrained accordingly. Here however the assumption is much less hard, and consists in computing the distance to the vertical chocked line as seen in Figure 6.

\subsection{Total orthogonal distance minimization}

Next stage in the parameterization procedure is to estimate the whole compressor model using orthogonal distance minimization. This requires including the efficiency model (14) which adds a new dimension to the problem and transfers the orthogonal projection subproblem to a three dimensional space, $\left(\bar{W}_{c}, \Pi_{c}, \eta_{c}\right)$. As it is already discussed in Section 4.2, the mass flow model is called inside the efficiency model to compute the desired outputs in a simulation framework. For this reason it is important to keep both models linked during the model parameterization to minimize the combined model errors.

First, the previous estimated parameters from the mass flow orthogonal estimation are used as initial guess for this stage of the algorithm. Then the implicit equation of the mass flow model (10) is extended with the efficiency coordinate

$$
\tilde{F}\left(\bar{W}_{c}, \Pi_{c}, \eta_{c}\right)=F\left(\bar{W}_{c}, \Pi_{c}\right)
$$

this will now represent a surface in the three dimensional space. Dropping the dependence on compressor speed, the 


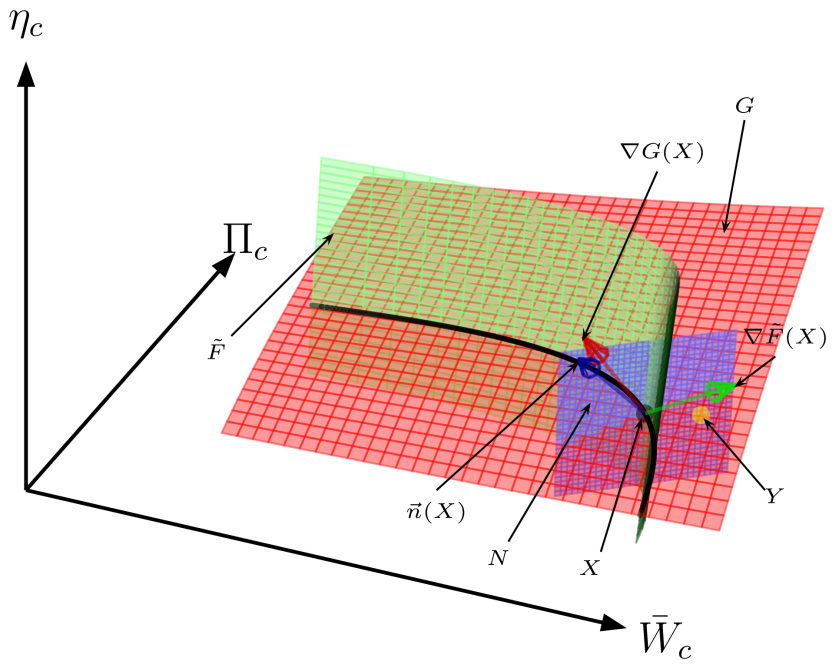

Fig. 7. Orthogonal projection sketch in $3 \mathrm{D}$ for a given measured point $(Y)$. The black solid curve defines the SpL, which is formed by the intersection of surfaces $G$ and $\tilde{F}$. The orthogonal plane $(N)$ is formed with the tangent vector to the $\mathrm{SpL}, \vec{n}(X)$, and the measurement point $(Y)$.

efficiency model is also rewritten in implicit form as

$$
G\left(\bar{W}_{c}, \Pi_{c}, \eta_{c}\right)=\eta_{c}-\frac{c_{p} T_{01}\left(\Pi_{c}^{(\gamma-1) / \gamma}-1\right)}{b-a \cdot \bar{W}_{c}}
$$

Together, $G$ and $\tilde{F}$ intersect in the space forming a curve that provides the relation between the three variables. This curve is the modeled $\mathrm{SpL}$ in the $\left(\bar{W}_{c}, \Pi_{c}, \eta_{c}\right)$ space. A representation of this intersection can be seen in Figure 7. It is important to note also that $G$ and $\tilde{F}$ depend on $\bar{N}_{c}$. However, the measured speed is considered accurate and thus given, this avoids adding a fourth dimension to the problem.

Following the same idea as in the two-dimensional case, the orthogonal projection has to be computed first by solving a system of equations. In the three-dimensional case and using again geometric equations, it looks as follows

$$
\left\{\begin{array}{l}
\tilde{F}(X)=0 \\
G(X)=0 \\
\vec{n}_{i}(X) \cdot\left(X_{i}-Y_{i}\right)+\vec{n}_{j}(X) \cdot\left(X_{j}-Y_{j}\right) \\
+\vec{n}_{k}(X) \cdot\left(X_{k}-Y_{k}\right)=0
\end{array}\right.
$$

where

$$
\begin{aligned}
& X=\left(\bar{W}_{c, \text { int }}, \Pi_{c, \text { int }}, \eta_{c, \text { int }}\right)^{T} \\
& Y=\left(\bar{W}_{c, \text { meas }}, \Pi_{c, \text { meas }}, \eta_{c, \text { meas }}\right)^{T}
\end{aligned}
$$

where in this case the points $X$ and $Y$ are augmented with the efficiency coordinate. The two first equations enforce the intersection point to be on the SpL model curve. The third equation forces the intersection point to belong to the plane formed by the tangent vector to the SpL curve and the measured point. The intersection of the planes in the space is represented in Figure 7 for a single measured point.

The normal vector of the orthogonal plane is constructed from the vectorial product of the gradients of $\tilde{F}$ and $G$ at the unknown intersection point $X=\left(\bar{W}_{c, \text { int }}, \Pi_{c, \text { inter }}, \eta_{c, \text { int }}\right)^{T}$

$$
\vec{n}(X)=\nabla \tilde{F}(X) \times \nabla G(X)
$$

Once the orthogonal intersection is computed, the vector of orthogonal distances for each measurement point is constructed using (22) accordingly, only extending the equation with the efficiency dimension.

As in the two-dimensional case, the measurement points in the shaded areas of Figure 6 are treated in the same way. The extension to the three dimensional case is to use (14) to compute the corresponding efficiency value. Furthermore the efficiency model introduces a new limitation, for measured points with $\Pi_{c, \text { meas }} \leq 1$. This is not an issue for any of the normal marine-size maps, neither for the automotive-size full map. Moreover, as when operating with pressure ratios smaller than one the compressor is working as a turbine, the modeled efficiency is limited to zero. In case there are measurements below unity, a possible approach is to compute the distances for the conflictive points $\left(\Pi_{c, \text { meas }} \leq 1\right)$, using the two dimensional algorithm presented in Subsection 5.2 and disregard the efficiency coordinate. Then the vector of residuals is formed as a combination of the output from both projection algorithms.

\subsection{Algorithm implementation}

The parameterization algorithm is implemented in MATLAB using the function $f$ solve to solve the othogonal projection equation systems at each least-squares iteration. The function lsqnonlin is used for solving the least-squares problem. The mass flow parameters are box constrained to prevent them from deviating too much from the initial guess for the Choke and Zero Slope lines.

Inside the parameter estimation process the compressor speed is normalized $\tilde{\bar{N}}=\bar{N} / \max (\bar{N})$ to avoid numerical problems with too distant numeric values of the parameters. Moreover, inside the orthogonal projection the space $\left(\bar{W}_{c}, \Pi_{c}, \eta_{c}\right)$ is also normalized. This is done to obtain a uniform shape of the geometric curves before solving the orthogonal projection system and thus avoid numerical problems. A common case is to obtain "deformed" SpL shapes due to the range of $\bar{W}_{c}$ being much bigger than the range of $\Pi_{c}$ for marine-size compressors. And the contrary for automotive-size compressors.

\section{Model Validation}

The model validation section is divided into two parts, first the results are analyzed for the automotive-size compressor from which there are measurements done at the low load area. Second the model performance is compared to two marine-size compressor maps. 


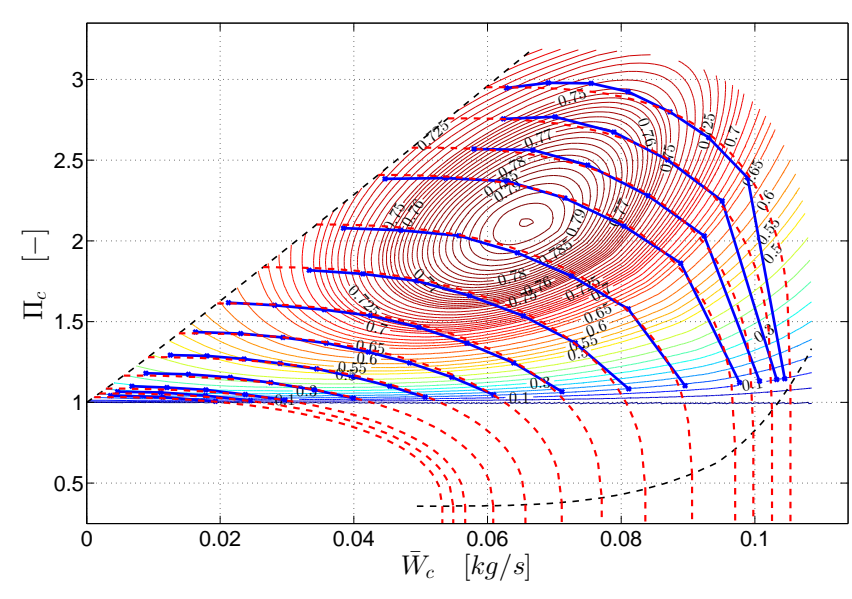

Fig. 8. GAR GT Compressor pressure ratio vs. mass flow. The dashed red lines are the modeled SpLs, the solid blue lines connect the measured points. The contour lines correspond to the efficiency values.

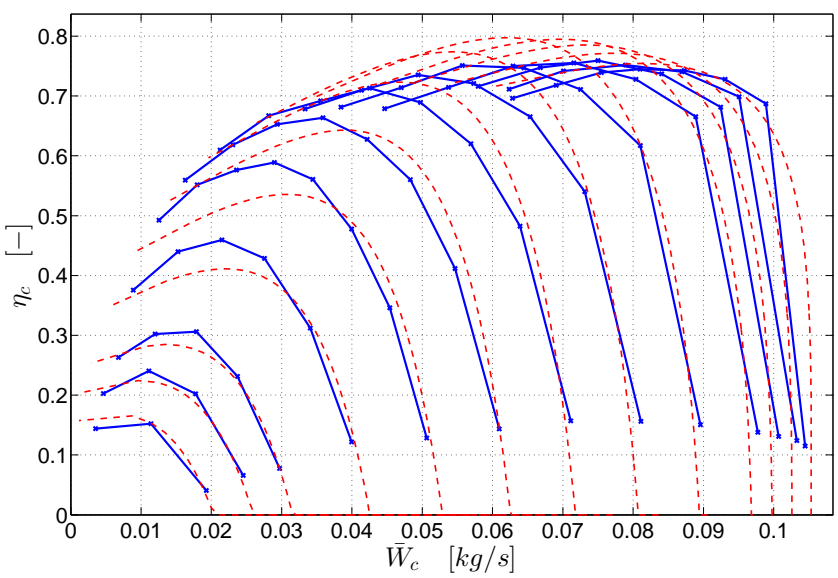

Fig. 9. GAR GT Compressor efficiency vs. mass flow. The dashed red lines are the modeled SpLs, the solid blue lines connect the measured points.

\subsection{Automotive-size Results}

Results for the full GAR GT map are shown in Figure 8 , where it is observed that the mass flow model is capable of predicting the measured points with high accuracy. Figure 9 shows the agreement for the modeled efficiency vs the mass flow, the general trend to lower SpLs is captured but the error is slightly bigger than for the $\bar{W}_{c}-\Pi_{c}$ case. Another observation is that the measured maximum efficiency is relatively flat around the mid-speed region. In this area the model predicts a slightly higher efficiency, a reason for this is that it has to have some curvature in this area to be able to match the efficiency drop for the lower SpLs. The relative errors of the model are shown in Table 1, where the largest error is in the efficiency dimension as it can be observed in Figure 9.
Table 1. Relative errors (\%) of the model vs the measurements for the GAR GT automotive-size compressor map. Mean indicates the mean value of the absolute error while Max. is the maximum relative error computed.

\begin{tabular}{c|c|c|}
\cline { 2 - 3 } & Mean & Max. \\
\hline $\bar{W}_{c}$ & 0.37 & 4.34 \\
$\Pi_{c}$ & 0.95 & 4.86 \\
$\eta_{c}$ & 5.70 & 14.45 \\
\hline
\end{tabular}

\subsection{Marine-size Results}

Figures 10 and 11 show the model agreement for the ABB VTR marine-size compressor map. Moreover the results for the ABB TPS map are depicted in Figures 12 and 13. If one compares the automotive-size compressor map to the two marine-size maps presented, the first difference observed is that the marine-size maps have a narrower shape. The choking line and the zero slope line are much closer than in the automotive case. Moreover the curvature of the ellipses representing the $\mathrm{SpL}$ is higher for the marine-size case, compare for example the SpLs in Figures 8 and 12. Nevertheless the model shows the capacity for adapting well to the particular shape of the marine-size compressors in the $\bar{W}_{c}-\Pi_{c}$ plane, see Figures 10 and 12. For the efficiency case the model also adapts quite well to the higher curvature of the SpLs in the $\eta_{c}-\bar{W}_{c}$ plane. However, as in the automotive case, it is not perfectly accurate to the maximum efficiency measured in each SpL in the mid-speed area, but the general trend is captured.

The errors using the total orthogonal distance minimization algorithm for all compressor maps, following the numbering from Figure 2, are presented in the white rows of Table 2 . In general the mean errors are below $2 \%$ and the maximum errors are not greater than $6 \%$. Table 2 also contains the model errors of the parameters before the total orthogonal distance minimization in the gray shadowed rows. Comparing the two sets of parameters one can observe that the total minimization step is important to decrease the errors of the efficiency coordinate. In particular the maximum error gets reduced substantially in all the marine maps studied. However, the errors for $\bar{W}_{c}$ and $\Pi_{c}$ tend to increase in this last step of the algorithm. This error increase is reasonable since the efficiency submodel and the mass flow submodel are linked, as discussed in Section 4. Note that the mass flow submodel is parameterized using the procedure described in Section 5.2, thus the errors are already low for $\bar{W}_{c}$ and $\Pi_{c}$. The total parameterization has to modify all nineteen parameters, so that the errors of the efficiency coordinate are reduced together with the errors of the other two coordinates. Hence this last step is important to balance the model accuracy along the three coordinates, $\left(\bar{W}_{c}, \Pi_{c}, \eta_{c}\right)$.

\section{Conclusions}

A control oriented, compact and continuous compressor model capable of extrapolating to low loads is presented. The mass flow model is based on an empirical model previously developed from a large database of automotive compressors. 
Table 2. Relative errors (\%) of the model vs the measurements for the marine-size compressor maps. Mean indicates the mean value of the absolute error while Max. is the maximum relative error computed. The white rows correspond to the errors after the total orthogonal distance minimization is done. The gray shadowed rows correspond to the errors before the total minimization.

\begin{tabular}{|c|c|c|c|c|c|c|c|c|c|c|c|c|c|c|c|c|c|c|}
\hline & \multicolumn{14}{|c|}{ Compressor No. } & \multicolumn{4}{|c|}{ Compressor } \\
\hline & \multicolumn{2}{|c|}{1} & \multicolumn{2}{|c|}{$\overline{2}$} & \multicolumn{2}{|c|}{$\overline{3}$} & \multicolumn{2}{|c|}{4} & \multicolumn{2}{|c|}{5} & \multicolumn{2}{|c|}{6} & \multicolumn{2}{|c|}{ r } & \multicolumn{2}{|c|}{$\overline{\text { TPS }}$} & \multicolumn{2}{|c|}{$\begin{array}{l}\text { VTR } \\
\end{array}$} \\
\hline & Mean & \begin{tabular}{|l} 
Max. \\
\end{tabular} & Mean & Max. & Mean & Max. & Mean & $\operatorname{Max}$ & Mean & $\operatorname{Max}$ & Mean & Max. & Mean & Max. & Mean & Max. & Mean & Max. \\
\hline$\overline{\bar{W}_{c}}$ & 0.28 & 1.46 & 0.65 & 2.45 & 0.47 & 3.42 & 0.72 & 2.49 & 0.92 & 4.25 & 0.76 & 2.73 & 1.28 & 3.34 & 0.79 & 2.98 & 0.56 & 2.25 \\
\hline$\Pi_{c}$ & 0.61 & 1.81 & 1.11 & 5.43 & 0.96 & 3.41 & 0.64 & 2.56 & 0.95 & 5.18 & 0.95 & 4.18 & 1.04 & 2.87 & 1.17 & 5.78 & 0.62 & 4.04 \\
\hline$\eta_{c}$ & 0.71 & 2.16 & 1.19 & 3.92 & 1.43 & 3.87 & 0.90 & 2.96 & 1.51 & 5.25 & 1.41 & 4.95 & 1.87 & 9.03 & 1.94 & 5.01 & 0.85 & 3.21 \\
\hline$\overline{\bar{W}_{c}}$ & 0.47 & 2.09 & 0.28 & 0.82 & 0.34 & 1.49 & 0.42 & 1.23 & 0.77 & 3.04 & 0.50 & 1.37 & 0.59 & 2.25 & 0.59 & 1.95 & 0.46 & 1.59 \\
\hline$\Pi_{c}$ & 0.46 & 1.84 & 0.76 & 2.17 & 0.88 & 2.07 & 0.54 & 1.59 & 0.86 & 2.66 & 0.72 & 1.87 & 0.74 & 2.51 & 1.00 & 3.61 & 0.64 & 2.31 \\
\hline$\eta_{c}$ & 1.04 & 7.41 & 2.51 & 13.33 & 2.85 & 12.84 & 1.39 & 7.16 & 2.24 & 9.76 & 2.87 & 12.78 & 1.67 & 14.27 & 2.72 & 10.32 & 1.46 & 5.83 \\
\hline
\end{tabular}

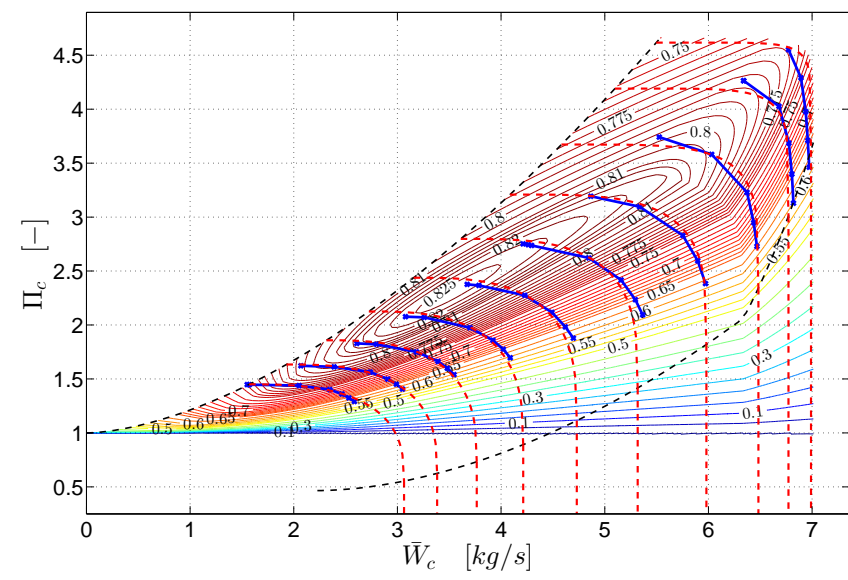

Fig. 10. ABB VTR compressor pressure ratio vs. mass flow. The dashed red lines are the modeled SpLs, the solid blue lines connect the measured points. The contour lines correspond to the efficiency values.

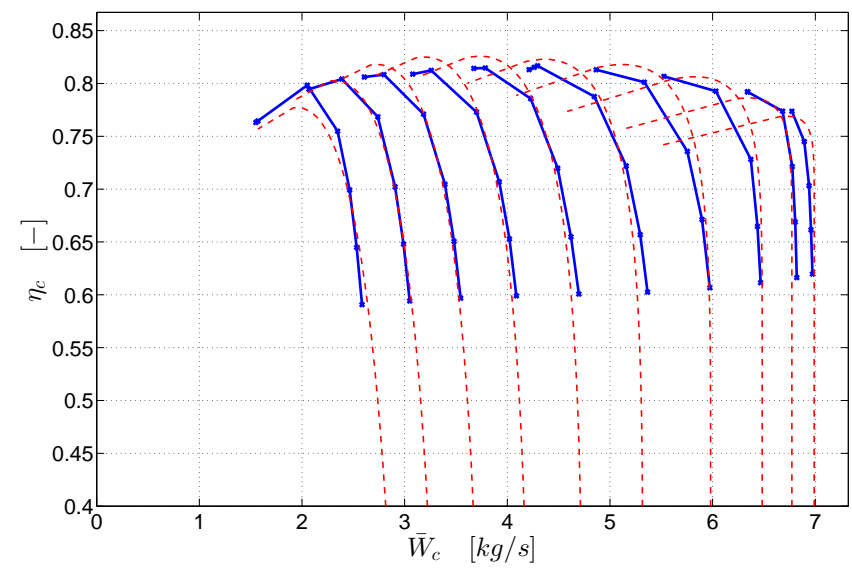

Fig. 11. ABB VTR compressor efficiency vs. mass flow. The dashed red lines are the modeled SpLs, the solid blue lines connect the measured points.

The approach consists of modeling each $\mathrm{SpL}$ as a super ellipse with its parameters dependent on compressor speed. It is shown here to adapt well to the marine-size compressor characteristics with the fifteen estimated parameters.

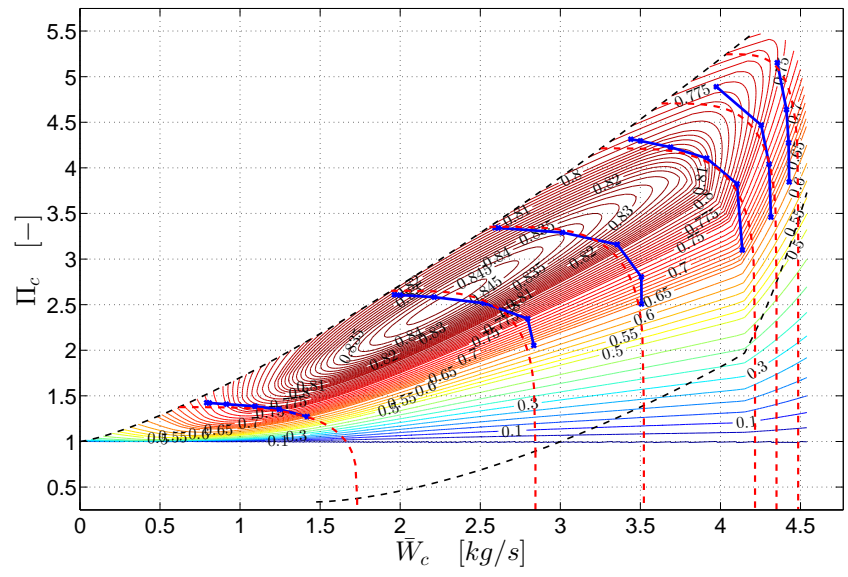

Fig. 12. ABB TPS compressor pressure ratio vs. mass flow. The dashed red lines are the modeled SpLs, the solid blue lines connect the measured points. The contour lines correspond to the efficiency values.

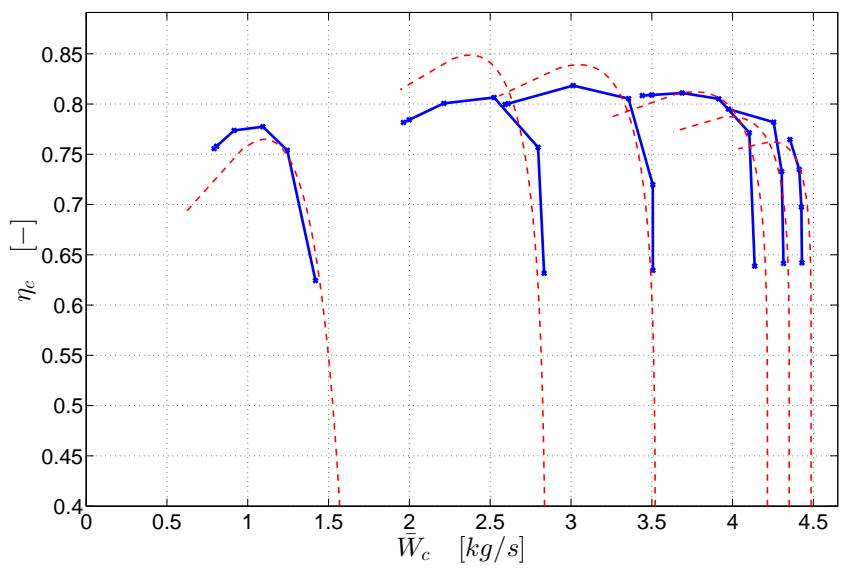

Fig. 13. ABB TPS compressor efficiency vs. mass flow. The dashed red lines are the modeled SpLs, the solid blue lines connect the measured points.

The efficiency model is based on Euler's equation for turbo machines. The adaptation of the physical equations leads to a rather simple model with four parameters that is capable of capturing the main characteristics of the efficiency 
for the measured SpLs and extrapolate to low speeds. The efficiency model cannot be properly validated at low loads due to the lack of measurements for marine-size compressors. Therefore this validation is done with data available from an automotive compressor, showing promising results. The weak points of the efficiency model and how to overcome them is also discussed, the price to pay is a more complex model with more parameters.

To simulate the model, the efficiency calculation requires the computed mass flow model. This means that the errors of the mass flow model will propagate to the efficiency model. Therefore a method for parameterizing both submodels at the same time is proposed. The parameterization method is based on minimizing the orthogonal distance between the modeled SpLs and the measurement points. To compute the orthogonal distance in an efficient way, the algorithm uses the analytical gradients of the model. The model is parameterized with nine different marine-size compressor maps showing a good agreement with the measurement data. Moreover, the results show that for the model to be accurate, it is important to parameterize both the mass flow and the efficiency submodels at the same time.

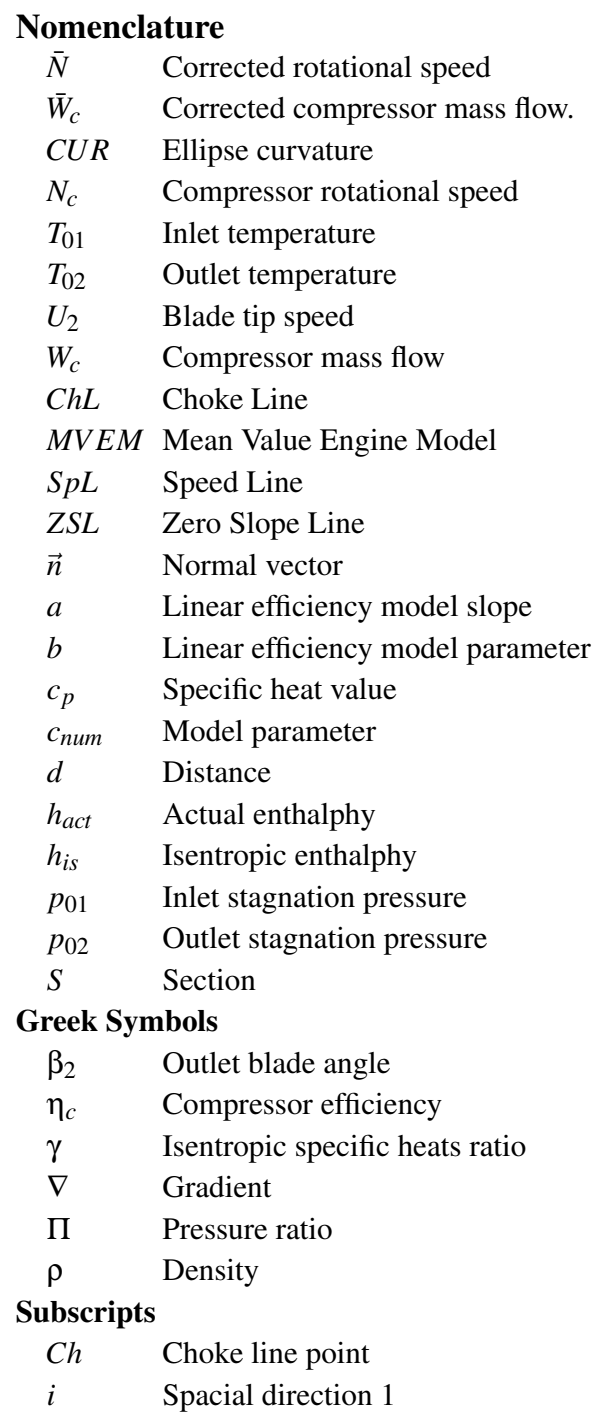

$\begin{array}{ll}\text { int } & \text { Intersection point } \\ j & \text { Spacial direction } 2 \\ k & \text { Spacial direction } 3 \\ \text { meas } & \text { Measured point } \\ Z S & \text { Zero slope line point }\end{array}$

\section{Acknowledgements}

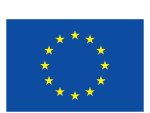

This project has received funding from the European Union's Horizon 2020 research and innovation programme under grant agreement No 634135.

ABB Turbo Systems Ltd. is greatly acknowledged for the discussions and for the permission to publish the compressor data. MAN Diesel \& Turbo is also acknowledged for their support and discussions about compressor modeling.

\section{References}

[1] International Maritime Organization, 2013. MARPOL: Annex VI and NTC 2008 with Guidelines for Implementation. IMO.

[2] Hendricks, E., 1986. "A compact, comprehensive model of a large turbocharged, two-stroke diesel engine". In SAE Technical Paper, SAE.

[3] Jensen, J.-P., Kristensen, A. F., Sorenson, S. C., Houbak, N., and Hendricks, E., 1991. "Mean value modeling of a small turbocharged diesel engine". In SAE Technical Paper, SAE, SAE International.

[4] Jung, M., 2003. "Mean-value modelling and robust control of the airpath of a turbocharged diesel engine". $\mathrm{PhD}$ thesis, Sidney Sussex College, Department of Engineering, University of Cambridge.

[5] Alegret, G., Llamas, X., Vejlgaard-Laursen, M., and Eriksson, L., 2015. "Modeling of a large marine twostroke diesel engine with cylinder bypass valve and EGR system". IFAC-PapersOnLine, 48(16), pp. 273 278. 10th IFAC Conference on Manoeuvring and Control of Marine Craft.

[6] Eriksson, L., 2007. "Modeling and control of turbocharged SI and DI engines". Oil \& Gas Science and Technology, 62(4), October, pp. 523-538.

[7] Moraal, P., and Kolmanovsky, I., 1999. “Turbocharger modeling for automotive control applications". In SAE Technical Paper, SAE International.

[8] Theotokatos, G., 2010. "On the cycle mean value modelling of a large two-stroke marine diesel engine". Proc Inst Mech Eng M: Journal of engineering for the maritime environment, 224(3), pp. 193-206.

[9] Karlsen, A. T., 2012. "On Modeling of a Ship Propulsion System for Control Purposes”. Master's thesis, NTNU, Trondheim.

[10] Hansen, J. M., Zander, C.-G., Pedersen, N., Blanke, M., and Vejlgaard-Laursen, M., 2013. "Modelling for control of exhaust gas recirculation on large diesel engines". 9th IFAC Conference on Control Applications in Marine Systems.

[11] Guan, C., Theotokatos, G., Zhou, P., and Chen, H., 
2014. "Computational investigation of a large containership propulsion engine operation at slow steaming conditions". Applied Energy, 130, pp. 370 - 383.

[12] Tsoutsanis, E., Meskin, N., Benammar, M., and Khorasani, K., 2015. "Transient gas turbine performance diagnostics through nonlinear adaptation of compressor and turbine maps". ASME J Gas Turb Pwr, 9(137).

[13] Watson, N., and Janota, M., 1982. Turbocharging the internal combustion engine. MacMillan, London.

[14] SAE J 922, 1995. Turbocharger Nomenclature and Terminology. SAE standard.

[15] Leufvén, O., and Eriksson, L., 2014. "Measurement, analysis and modeling of centrifugal compressor flow for low pressure ratios". Int. J. Engine Res., December, pp. 1-16.

[16] Leufvén, O., and Eriksson, L., 2013. "A surge and choke capable compressor flow model - validation and extrapolation capability". Control Eng. Pract., 21(12), December, pp. 1871-1883.

[17] Martin, G., Talon, V., Higelin, P., Charlet, A., and Caillol, C., 2009. "Implementing turbomachinery physics into data map-based turbocharger models". SAE Int. J. of Engines, 2(1), April, pp. 211-229.

[18] Sorenson, S. C., Hendricks, E., Magnusson, S., and Bertelsen, A., 2005. "Compact and accurate turbocharger modelling for engine control”. In SAE Technical Paper, SAE International.

[19] El Hadef, J., Colin, G., Chamaillard, Y., and Talon, V., 2012. "Physical based algorithms for interpolation and extrapolation of turbocharger data maps". SAE Int. J. of Engines, 5(2), April, pp. 363-378.

[20] Casey, M., and Schlegel, M., 2009. "Estimation of the performance of turbocharger compressors at extremely low pressure ratios". Proc Inst Mech Eng A: Journal of Power and Energy, 2(224), November, pp. 239-250.

[21] Casey, M., and Robinson, C., 2012. "A method to estimate the performance map of a centrifugal compressor stage.”. ASME J Turbomach, 2(135).

[22] Casey, M., and Rusch, D., 2014. "The matching of a vaned diffuser with a radial compressor impeller and its effect on the stage performance". ASME J Turbomach, 12(136).

[23] Eriksson, L., and Nielsen, L., 2014. Modeling and Control of Engines and Drivelines. John Wiley \& Sons.

[24] Dixon, S. L., and Hall, C. A., 2013. Fluid Mechanics and Thermodynamics of Turbomachinery, 7th ed. Butterworth-Heinemann.

[25] Nocedal, J., and Wright, S. J., 2006. Numerical Optimization, 2nd ed. Springer.

[26] Gander, W., Golub, G. H., and Strebel, R., 1994. "Least-squares fitting of circles and ellipses". BIT, 34, pp. 558-578.

[27] Ahn, S. J., Rauh, W., and Warnecke, H.-J., 2001. "Least-squares orthogonal distances fitting of circle, sphere, ellipse, hyperbola, and parabola". Pattern Recognition, 34, pp. 2283-2303.

[28] Boggs, P. T., H-Byrd, R., and Schnabel, R. B., 1987. “A stable and efficient algorithm for nonlinear orthogonal distance regression". SIAM J. Sci. Stat. Comput., 8(6), pp. 1052-1078.

\section{Appendix A: Model Gradients}

The analytic gradients are used in the algorithm. With a fixed compressor speed and defining the coordinates $X=$ $\left(\bar{W}_{c}, \Pi_{c}, \eta_{c}\right)^{T}$, the gradient of the mass flow model is

$$
\begin{aligned}
\nabla \tilde{F}(X) & =\left(\frac{\mathrm{d} \tilde{F}(X)}{\mathrm{d} \bar{W}_{c}}, \frac{\mathrm{d} \tilde{F}(X)}{\mathrm{d} \Pi_{c}}, \frac{\mathrm{d} \tilde{F}(X)}{\mathrm{d} \eta_{c}}\right)^{T} \\
\frac{\mathrm{d} \tilde{F}(X)}{\mathrm{d} \bar{W}_{c}} & =\frac{C U R}{\Pi_{Z S}-\Pi_{C h}}\left(\frac{\Pi_{c}-\Pi_{C h}}{\Pi_{Z S}-\Pi_{C h}}\right)^{C U R-1} \\
\frac{\mathrm{d} \tilde{F}(X)}{\mathrm{d} \Pi_{c}} & =\frac{C U R}{\bar{W}_{C h}-\bar{W}_{Z S}}\left(\frac{\bar{W}_{c}-\bar{W}_{Z S}}{\bar{W}_{C h}-\bar{W}_{Z S}}\right)^{C U R-1} \\
\frac{\mathrm{d} \tilde{F}(X)}{\mathrm{d} \eta_{c}} & =0
\end{aligned}
$$

For the two dimensional case $(F)$, the gradient is formed with (30) and (31). The efficiency model gradient is computed as

$$
\begin{aligned}
\nabla G(X) & =\left(\frac{\mathrm{d} \tilde{F}(X)}{\mathrm{d} \bar{W}_{c}}, \frac{\mathrm{d} \tilde{F}(X)}{\mathrm{d} \Pi_{c}}, \frac{\mathrm{d} \tilde{F}(X)}{\mathrm{d} \eta_{c}}\right)^{T} \\
\frac{\mathrm{d} G(X)}{\mathrm{d} \bar{W}_{c}} & =-\frac{a c_{p} T_{01}\left(\Pi_{c}^{(\gamma-1) / \gamma}-1\right)}{\left(b-a \cdot \bar{W}_{c}\right)^{2}} \\
\frac{\mathrm{d} G(X)}{\mathrm{d} \Pi_{c}} & =-\frac{c_{p} T_{01}(1-\gamma)}{\left(b-a \cdot \bar{W}_{c}\right) \gamma} \Pi_{c}^{-1 / \gamma} \\
\frac{\mathrm{d} G(X)}{\mathrm{d} \eta_{c}} & =1
\end{aligned}
$$

Then the normal vector for the $3 \mathrm{D}$ case projection, defined in (28) has the following expression

$$
\begin{gathered}
\vec{n}(X)=\nabla \tilde{F}(X) \times \nabla G(X)=\left|\begin{array}{ccc}
\vec{i} & \vec{j} & \vec{k} \\
\frac{\mathrm{d} \tilde{F}(X)}{\mathrm{d} \bar{W}_{c}} & \frac{\mathrm{d} \tilde{F}(X)}{\mathrm{d} \Pi_{c}} & \frac{\mathrm{d} \tilde{F}(X)}{\mathrm{d} \eta_{c}} \\
\frac{\mathrm{d} G(X)}{\mathrm{d} \bar{W}_{c}} & \frac{\mathrm{d} G(X)}{\mathrm{d} \Pi_{c}} & \frac{\mathrm{d} G(X)}{\mathrm{d} \eta_{c}}
\end{array}\right|=(37) \\
\left(\frac{\mathrm{d} \tilde{F}(X)}{\mathrm{d} \Pi_{c}},-\frac{\mathrm{d} \tilde{F}(X)}{\mathrm{d} \bar{W}_{c}}, \frac{\mathrm{d} \tilde{F}(X)}{\mathrm{d} \bar{W}_{c}} \cdot \frac{\mathrm{d} G(X)}{\mathrm{d} \Pi_{c}}-\frac{\mathrm{d} \tilde{F}(X)}{\mathrm{d} \Pi_{c}} \cdot \frac{\mathrm{d} G(X)}{\mathrm{d} \bar{W}_{c}}\right)^{T}
\end{gathered}
$$

